\title{
Effect of Aspect Ratio on Laminar Natural Convection in Partially Heated Enclosure
}

\author{
Alireza Falahat \\ Department of Mechanics, Mahshahr Branch, Islamic Azad University, Mahshahr, Iran \\ *Corresponding Author: a.falahat@mahshariau.ac.ir
}

Copyright (C) 2014 Horizon Research Publishing All rights reserved.

\begin{abstract}
In this study, the influence of aspect ratio and Rayleigh number have been studied for a square cavity. The constant partial cooling at left vertical wall and partial heating at the right vertical wall along with the adiabatic top and bottom walls of cavity filled with the water. The aspect ratio was varied from 0.5 to 4 . The governing equations are solved with finite volume approach for the range of Rayleigh number as 1000 to 1000000 . Results have clearly indicated Heat transfer enhances with increasing of Rayleigh number for the whole of aspect ratio. The results show the Nusselt number increases from aspect ratio 0.5 to 1 and beyond that it decreases smoothly.
\end{abstract}

Keywords Laminar Natural Convection, Aspect Ratio, Finite Volume Method, Cavity

\section{Introduction}

Natural convection phenomena in rectangular or square cavity has received considerable attention in the last four decades due to its wide application in the area of engineering.

The studies on natural convection in enclosure focus on mainly differentially heated enclosure in the literature. Many study related to this subject is available in the study of Vahl Davis [1] and Vahl Davis and Jones [2]. Chu et al. [3] studied the natural convection in an enclosure with a partial heater located to the left vertical wall and the enclosure cooled from right vertical wall. They investigated the problem both numerically and experimentally. Khanafer et al.[4] numerically investigated the natural convection heat transfer of a copper-water nanofluid in a two-dimensional enclosure. They showed that the heat transfer rate increases with an increase in the volumetric fraction at any given Rayleigh number.

laminar natural convection in cavity heated from below and cooled from above for a wide variety of thermal boundary conditions at the sidewalls has been carried numerically by Corcione [5].
Varol et al. [6] analyzed numerically natural convection heat transfer from a protruding heater located in a triangular enclosure.

Bairi [7] studied numerically and experimentally the natural convection in 2D, titled, square cavity filled air. He presented the Nusselt- Rayleigh correlations for different inclination angle of the cavity.

The main objective of this investigation is to analyze the effects of aspect ratio on natural convection in a square cavity with constant partial cooling at left vertical wall and partial heating at the right vertical wall along with the adiabatic top and bottom walls is studied numerically using the finite volume method. In the present study we examine the heat transfer and fluid flow for different aspect ratio and different Rayleigh number in a cavity.

\section{Mathematical Formulation}

The physical model and the coordinate system considered in the investigation are shown in Figure 1. The system was considered to be incompressible, steady-state, Newtonian and the Boussinesq approximation was applied for fluid with constant physical properties. It is assumed that the radiation effect can be taken to be negligible. The gravitational acceleration acts in the negative y-direction.

By assimilating the above assumptions, the system of the governing equations can be expressed

Continuity equation

$$
\frac{\partial u}{\partial x}+\frac{\partial v}{\partial y}=0
$$

Momentum equation

$$
\begin{aligned}
& u \frac{\partial u}{\partial x}+v \frac{\partial u}{\partial y}=\frac{1}{\rho}\left[\mu\left(\frac{\partial^{2} u}{\partial x^{2}}+\frac{\partial^{2} u}{\partial y^{2}}\right)-\frac{\partial p}{\partial x}\right] \\
& u \frac{\partial v}{\partial x}+v \frac{\partial v}{\partial y}=\frac{1}{\rho}\left[\begin{array}{c}
-\frac{\partial p}{\partial y}+\mu\left(\frac{\partial^{2} v}{\partial x^{2}}+\frac{\partial^{2} v}{\partial y^{2}}\right)+ \\
(\rho \beta) g\left(T-T_{c}\right)
\end{array}\right]
\end{aligned}
$$

Energy equation 


$$
u \frac{\partial T}{\partial x}+v \frac{\partial T}{\partial y}=\alpha\left(\frac{\partial^{2} T}{\partial x^{2}}+\frac{\partial^{2} T}{\partial y^{2}}\right)
$$

where $u$ and $v$ are the velocity components in the $x$ and $y$ directions, respectively. $p$ is the pressure, $T$ is the temperature and $\beta$ is the thermal expansion coefficient.

The above equations can be converted to nondimensional forms using the following nondimensional parameters.

$$
\begin{aligned}
& X=\frac{x}{L} \quad Y=\frac{y}{L} \\
& U=\frac{u L}{\alpha} \quad V=\frac{v L}{\alpha} \\
& P=\frac{p L^{2}}{\rho \alpha^{2}} \quad \theta=\frac{T-T_{c}}{T_{h}-T_{c}} \\
& R a=\frac{g \beta L^{3}\left(T_{h}-T_{c}\right)}{\alpha v} \quad \operatorname{Pr}=\frac{v}{\alpha}
\end{aligned}
$$

The governing equations are written in the following dimensionless form:

$$
\begin{aligned}
& \frac{\partial U}{\partial X}+\frac{\partial V}{\partial Y}=0 \\
& U \frac{\partial U}{\partial X}+V \frac{\partial U}{\partial Y}=-\frac{\partial P}{\partial X}+ \\
& \frac{\mu}{\rho \alpha}\left(\frac{\partial^{2} U}{\partial X^{2}}+\frac{\partial^{2} U}{\partial Y^{2}}\right) \\
& U \frac{\partial V}{\partial X}+V \frac{\partial V}{\partial Y}=-\frac{\partial P}{\partial Y}+ \\
& \frac{\mu}{\rho \alpha}\left(\frac{\partial^{2} V}{\partial X^{2}}+\frac{\partial^{2} V}{\partial Y^{2}}\right)+R a \operatorname{Pr} \theta \\
& U \frac{\partial \theta}{\partial X}+V \frac{\partial \theta}{\partial Y}=\left(\frac{\partial^{2} \theta}{\partial X^{2}}+\frac{\partial^{2} \theta}{\partial Y^{2}}\right)
\end{aligned}
$$

The dimensionless boundary conditions of the problem under consideration can be written as

$$
\begin{aligned}
& U=V=0 \quad \text { at all walls } \\
& \frac{\partial \theta}{\partial Y}=0 \quad \text { at } \quad Y=0 \text { and } A R \\
& \theta(0, Y)=0 \quad \text { when } \quad \frac{A R}{2} \leq Y \leq A R \\
& \frac{\partial \theta}{\partial X}=0 \quad \text { when } \quad 0 \leq Y \leq \frac{A R}{2} \\
& \theta(1, Y)=1 \quad \text { when } \quad 0 \leq Y \leq \frac{A R}{2} \\
& \frac{\partial \theta}{\partial X}=0
\end{aligned}
$$

The average Nusselt number $(N u)$ at right side wall is determined by integrating $\mathrm{Nu}$ along the hot wall.

$$
N u=\frac{1}{A R} \int_{0}^{\frac{A R}{2}} N u(Y) d Y
$$

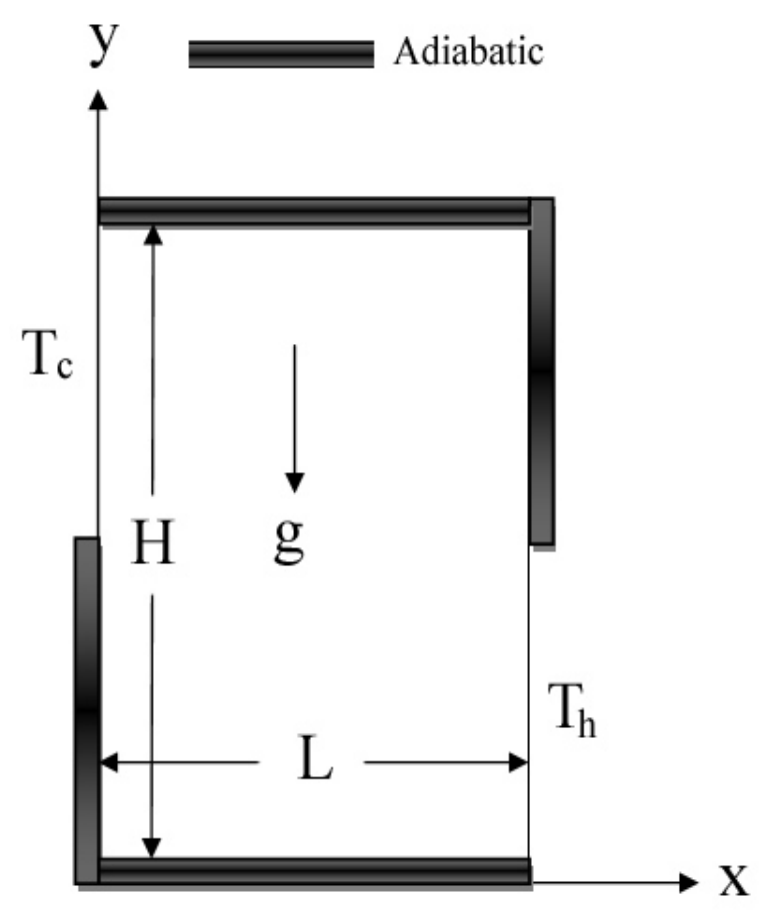

Figure 1. Schematic diagram of the physical system

\section{Numerical Scheme}

The numerical solution is obtained using a commercial CFD software, Fluent, which employs a finite volume method for the discretization of the continuity, momentum and energy equations. The pressure-based solver is adopted which was developed for low-speed incompressible flows. Owing to this technique the governing equations are solved sequentially (i.e., segregated from one another). Because the governing equations are non-linear and coupled, the solution loop must be carried out iteratively in order to obtain a converged numerical solution. The SIMPLE algorithm is used to couple the pressure and velocity terms. Discretization of the momentum and energy equations is performed by a second order upwind scheme and pressure interpolation is provided by PRESTO scheme [8]. Convergence criterion considered as residuals is admitted $10^{-3}$ for momentum and continuity equations and for the energy equation it is lower than $10^{-6}$.

\section{Grid Independent Study and Validation}

In this study we have used a uniform grid mesh at the walls; see Figure 2 for $\mathrm{AR}=1$, to allow grid-independent 
examination, the numerical procedure has been conducted for different grid resolutions.

Figure 3 shows the influence of the number of the grid points for pure water with $R a=10^{5}$ and $\mathrm{AR}=1$ of fluid confined within the present configuration. From this figure it is clear that the grid system of $81 \times 81$ is fine enough to obtain accurate results. We therefore adopted a grid system of $81 \times 81$. The validation of the adopted results has already conducted by authors who are available in Figure 4, in this figure, working fluid is air, the agreement is good. These tests provide a strong validation of our numerical results.

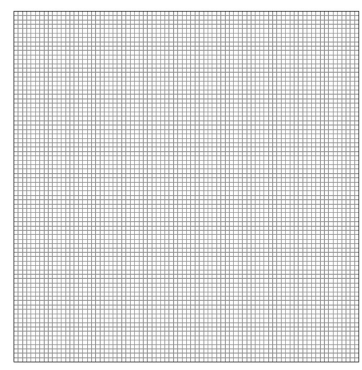

Figure 2. Uniform grid structure used in the computations for $\mathrm{AR}=1$

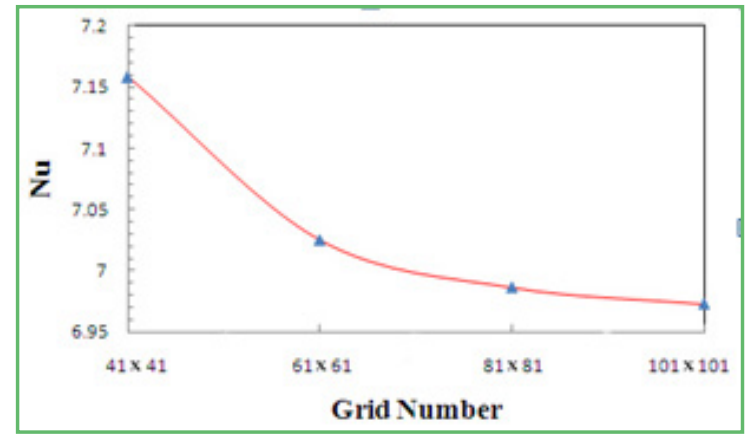

Figure 3. Grid point independence study for $R a=10^{5}$ and $\mathrm{AR}=1$

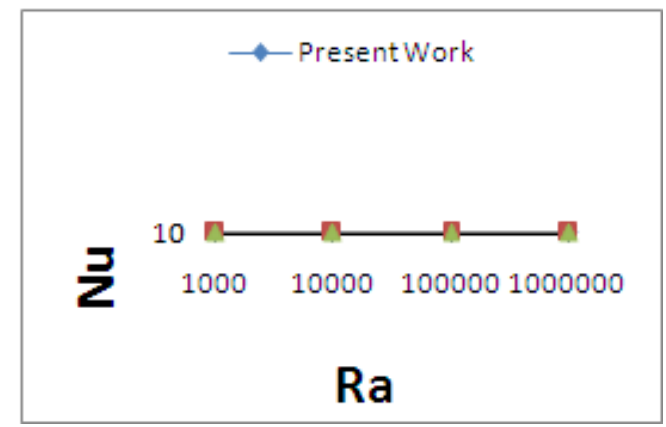

Figure 4. Validation of the present work against with other published works

\section{Results}

Numerical analysis of buoyancy induced flow in a rectangular cavity with constant partial cooling at left vertical wall and partial heating at the right vertical wall along with the adiabatic top and bottom walls filled with the water is studied numerically using the finite volume method.
The effect of aspect ratio and Rayleigh number are analyzed.

Figure 5 shows the effect of aspect ratio on streamlines and isotherms. As shown in the figure, the streamlines is rotating counter clockwise consequently the region near to the heated and cooled vertical wall (conduction zone) is becomes vertical line, this indicates that the buoyancy forces are able to overcome the retarding influence of the viscous forces. A single counter clockwise rotating cell is found for all aspect ratios. The cell is skewed upward along the left (cold) wall and downward along the right (hot) wall. The streamline contours show enhancements in the values of the maximum stream function. For $\mathrm{AR}=1$ shows that the stratified temperature in the core region pushes the flow along the adiabatic walls to the side and with increasing aspect ratio, Isotherm couture's show that the counter clockwise rotation of the cell in the core region is accelerated, bending the isotherms in the core region such that they are no longer orthogonal to the gravitational field. This distortion of the temperature field is due to the increase in speed of the counter clockwise rotating cell.

Figure 6 shows a streamlines and isotherms using different values of Rayleigh number for $\mathrm{AR}=1$. For $R a=10^{3}$, a central circulation cell is observed within the enclosure as a dominant characteristic of the flow field. However, as Rayleigh number increases the circulation zone is changed to an elliptical pattern $\left(R a=10^{4}\right)$ and eventually is broken up into more circulation zones $\left(R a=10^{6}\right)$. In addition, increasing Ra results in higher intensity of streamlines near the hot and cold walls. The corresponding isotherms show that the Rayleigh number increases a more important role in the formation of the isotherms. When, Rayleigh number increases, the thermal boundary layer near the walls becomes thinner, indicating a higher heat transfer rate as Rayleigh number increases.

Fig. 7 shows the variation of average Nusselt number with aspect ratio for different Rayleigh number. Average Nusselt number is increased with increasing of Rayleigh number monotonically. It is demonstrated that the case of aspect ratio of 1 has the highest Nusselt number due to the low velocity encountered at this aspect ratio; see Fig. 8, where the vertical velocity is plotted at the middle of the heated wall $(\mathrm{Y}=0.5)$ for $R a=10^{5}$. Also, Fig. 6 shows the maximum Nusselt number occurs for the case of aspect ratio of 1 . The Nusselt number is increases as the aspect ratio AR increases from 0.5 to 1 and beyond that it is decreases smoothly.

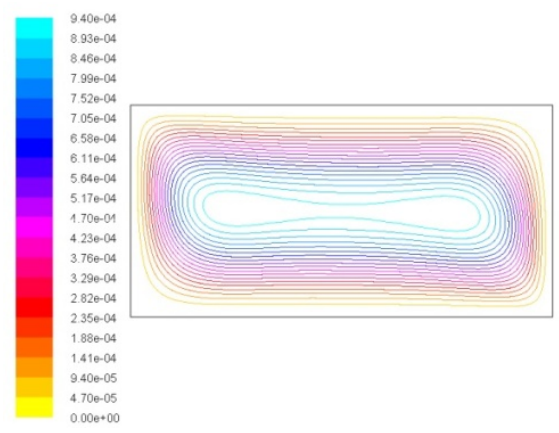

$\mathrm{AR}=0.5$ 


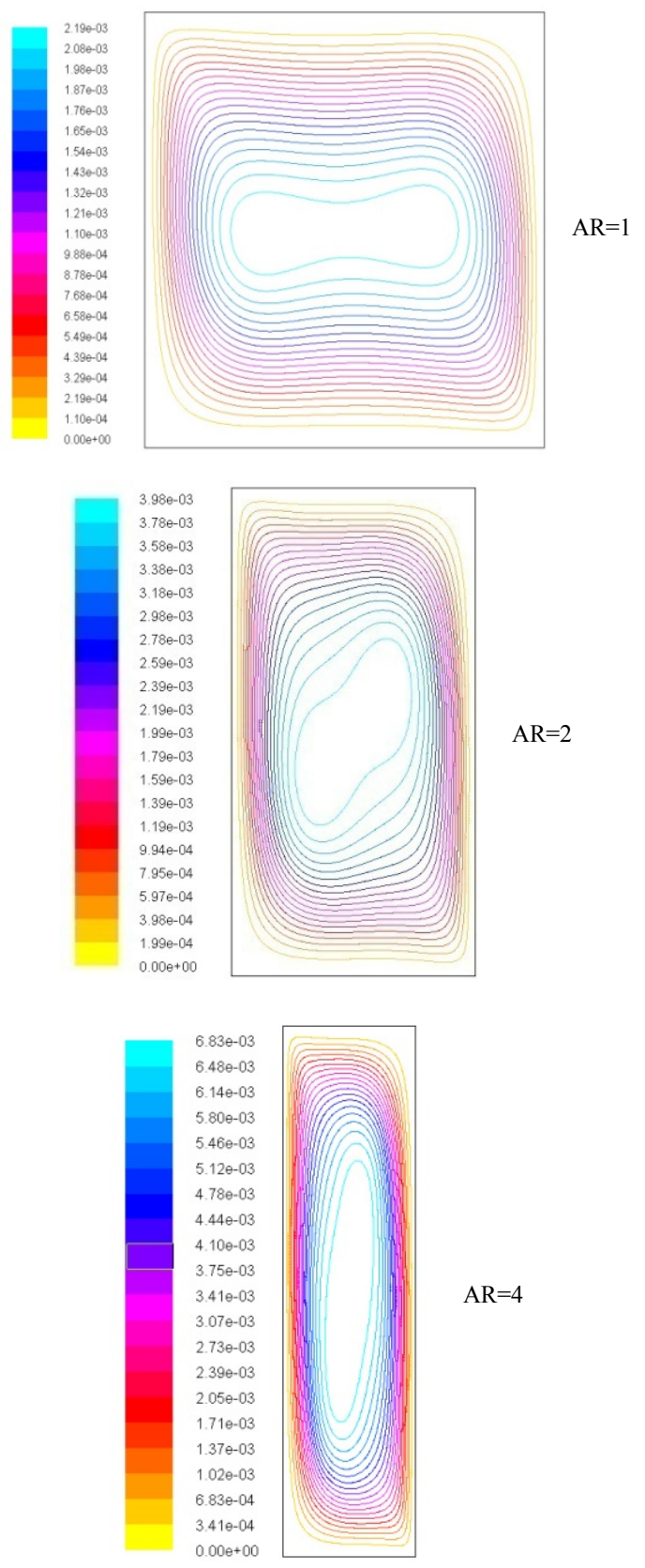

Figure 5a. Comparison of the Streamlines at $R a=10^{5}$ for various aspect ratio

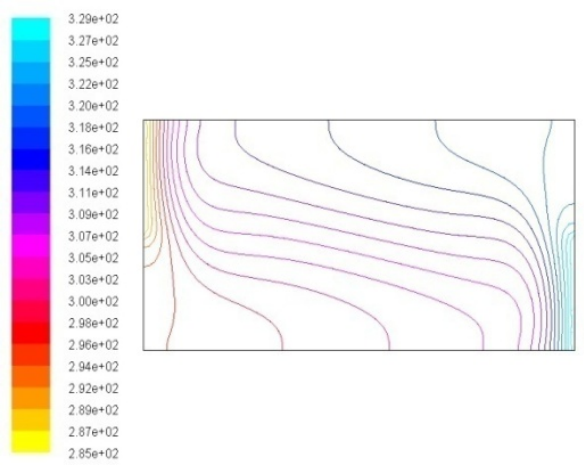

$$
\mathrm{AR}=0.5, \psi_{\max }=0.000893
$$

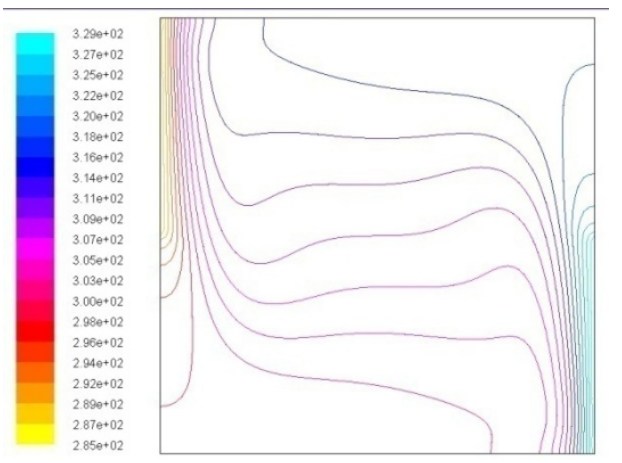

$$
\mathrm{AR}=1, \quad \psi_{\max }=0.002085
$$

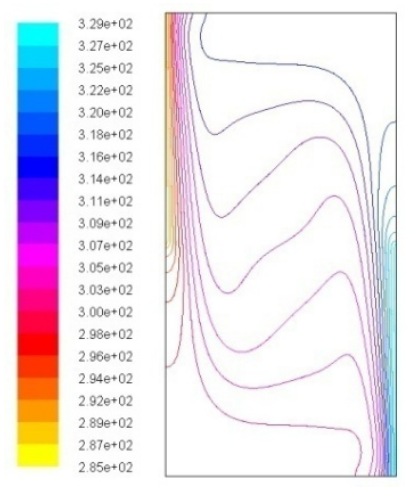

$\mathrm{AR}=2, \quad \psi_{\max }=0.003779$

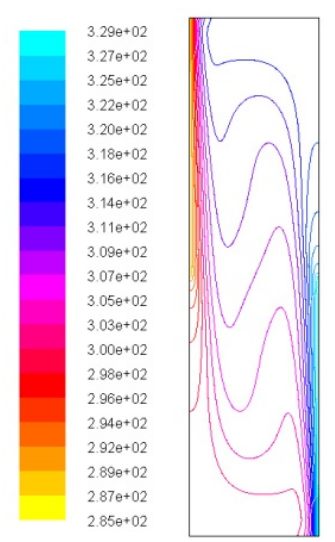

$\mathrm{AR}=4, \quad \psi_{\max }=0.006484$

Figure 5b. Comparison of the isotherms at $R a=10^{5}$ for various aspect ratio

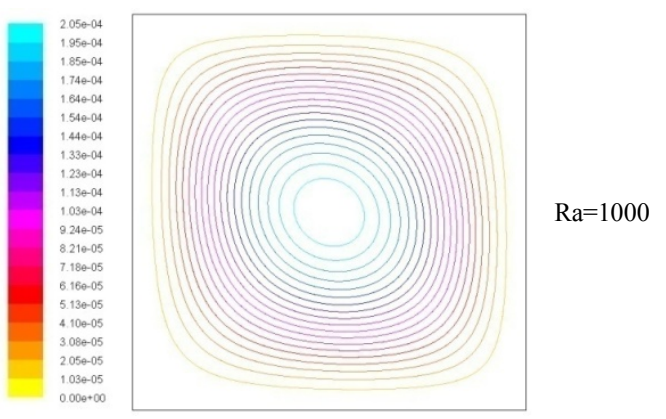




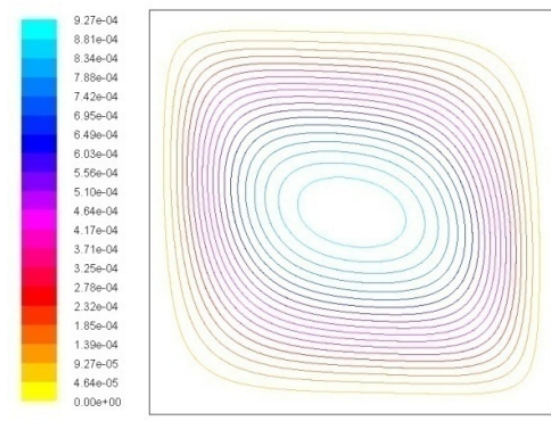

$\mathrm{Ra}=10000$

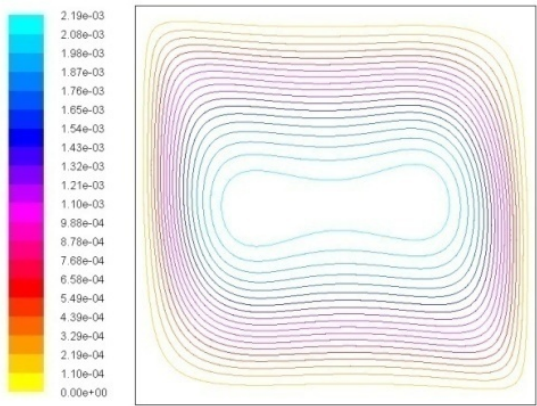

$\mathrm{Ra}=100000$

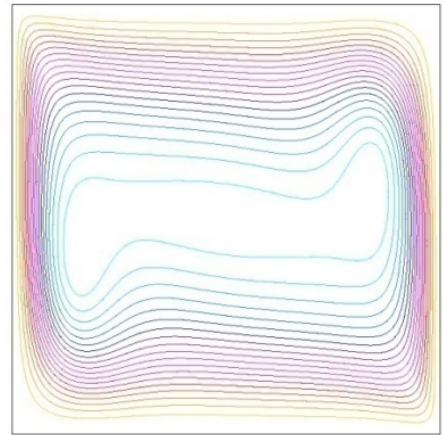

Figure 6a. Streamlines for $\mathrm{AR}=1$
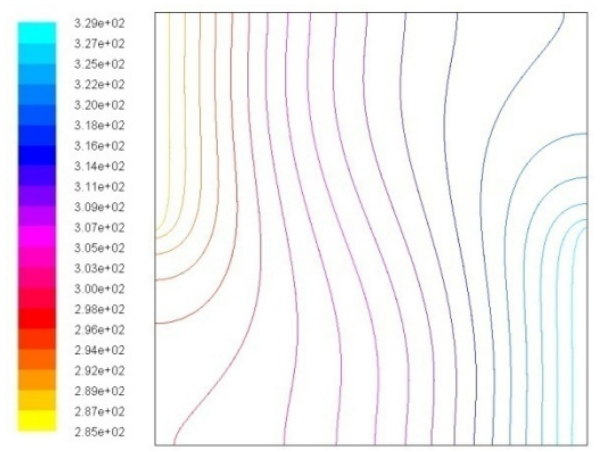

$\mathrm{Ra}=1000$
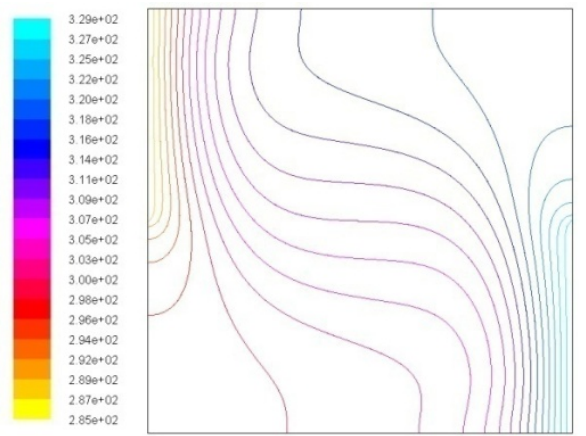

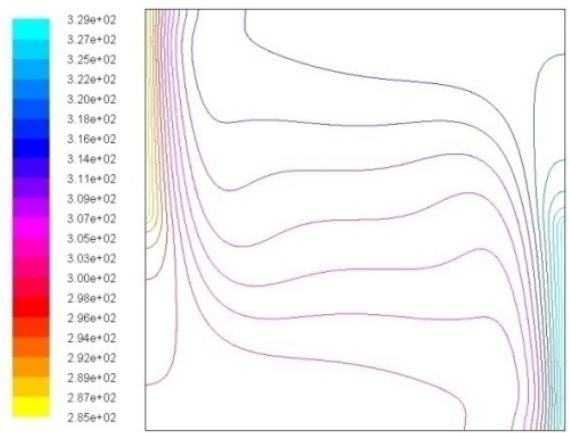

$\mathrm{Ra}=100000$

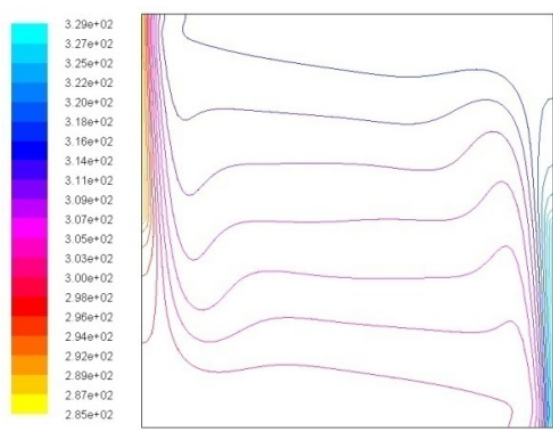

$\mathrm{Ra}=1000000$

Figure 6b. Isotherms for $\mathrm{AR}=1$

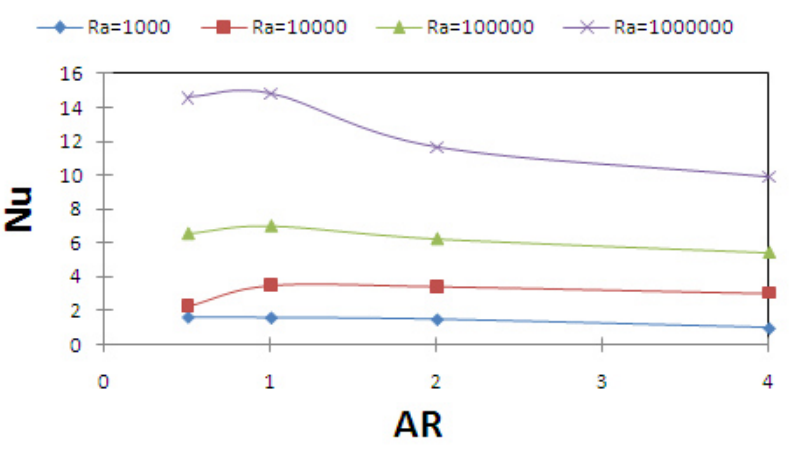

Figure 7. Effect of aspect ratio on average Nusselt number at various Rayleigh number

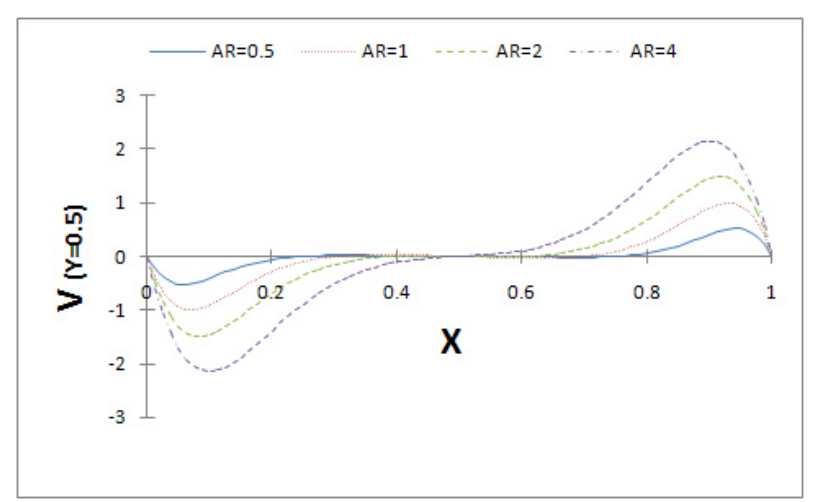

$\mathrm{Ra}=10000$

Figure 8. Variation of $y$-kelocity at the midsection of cavity for different aspect ratio and $R a=10$

\section{Conclusion}

In this paper the influence of aspect ratio and Rayleigh 
number have been studied for a square cavity with constant partial cooling at left vertical wall and partial heating at the right vertical wall along with the adiabatic top and bottom walls filled with the water. The results of the numerical analysis lead to the following conclusions.

Results have clearly indicated Heat transfer enhances with increasing of Rayleigh number. For low Rayleigh numbers, where the conduction heat transfer dominates, similar flow and temperature patterns are obtained for different aspect ratio in the range of $\mathrm{AR}=0.5,1,2,4$. However, as Rayleigh number increases positioning the enclosure at different aspect ratio affect the formation of flow and temperature patterns. The most enhancements belongs to aspect ratio $A R=1$. The aspect ratio of $A R=1$ has the highest Nusselt number due to the low velocity encountered at this aspect ratio and the minimum Nusselt number occurs for the case of aspect ratio of 4 .

\section{REFERENCES}

[1] G. Vahl Davis, Natural convection of air in a square cavity: a benchmark numerical solution, Int. J. Numer. Methods Fluids, Vol. 3, 249-264, 1983.
[2] G. Vahl Davis, J.P. Jones, Natural convection in a square cavity: a comparison study, Int. J. Numer. Methods Fluids, Vol. 3, 227-248, 1983.

[3] H.H.S. Chu, S.W. Churchill, C.V.S. Patterson, The effect of heater size, location, aspect ratio, and boundary conditions on two-dimensional, laminar, natural convection in rectangular channels, J. Heat Transfer, Vol. 98, 194-201, 1976.

[4] K. Khanafer, K. Vafai, and M. Lightstone, Buoyancy-Driven Heat Transfer Enhancement in a Two-Dimensional Enclosure Utilizing Nanofluids, Int. J. Heat Mass Transfer, Vol.46, 3639-3653, 2003.

[5] M. Corcione. Effects of the thermal boundary conditions at the sidewalls upon natural convection in rectangular enclosures heated from bellow and cooled from above. Int $\mathrm{J}$ Therm Sci, Vol. 42, 199-208, 2003.

[6] Y. Varol, H.F. Oztop, T. Yilmaz, Natural convection in triangular enclosures with protruding isothermal heater, Int. J. Heat Mass Transfer, Vol. 50, 2451-2462, 2007.

[7] Bairi, A., "Nusselt-Rayleigh correlations for design of industrial elements: Experimental and numerical investigation of natural convection in tilted square air filled enclosures", Energy Conversion and Management, Vol. 49, No. 4, (2008), 771-782.

[8] Fluent User's Guide, Release 6.3.26, Fluent Incorporated,2005. 